\title{
COMPASS results on the transverse spin asymmetry in identified dihadron production in SIDIS
}

\author{
Christopher Braun* \\ on behalf of the COMPASS collaboration \\ Physikalisches Institut IV of the University Erlangen-Nürnberg (FAU) \\ Erwin-Rommel-Strasse 1, 91058 Erlangen, Germany \\ E-mail: christopher.braunecern.ch
}

\begin{abstract}
The parton distribution function $h_{1}^{q}(x)$ of a transversely polarized quark $q$ inside a transversely polarized nucleon, is chiral-odd and therefore not accessible in inclusive deep-inelastic scattering. It can however be observed in semi-inclusive deep-inelastic scattering (SIDIS) in combination with another chiral-odd function, for instance the dihadron interference fragmentation function (DiFF) $H_{1}^{\triangleleft q}$. The $160 \mathrm{GeV} / c$ polarized muon beam of CERN's M2 beamline allows COMPASS to investigate transverse spin effects using polarized solid-state targets. In this contribution an overview of COMPASS results for the azimuthal asymmetry in identified dihadron production is given. Pions and kaons were identified by a RICH detector. Recently, the full set of this asymmetry from the COMPASS data on the deuteron and the proton target became available. All data, including early data sets, were analyzed using the same methods and requirements. The results are compared to model predictions and HERMES data. Furthermore an point-by-point extraction of the Transversity distribution function $h_{1}(x)$ for $u$ and $d$ quarks was carried out with this full data set.
\end{abstract}

XXII. International Workshop on Deep-Inelastic Scattering and Related Subjects, 28 April - 2 May 2014

Warsaw, Poland

${ }^{*}$ Speaker. 


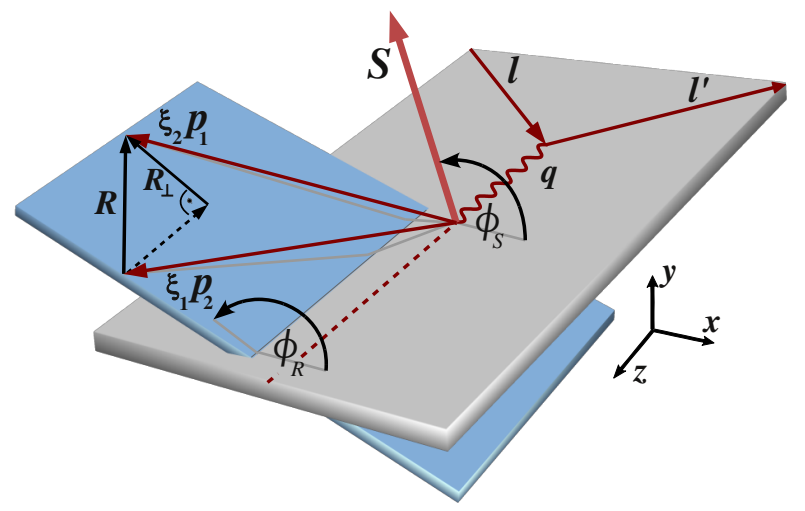

Figure 1: Simplified scheme of the dihadron production process: The incoming lepton and scattered lepton with their three-momenta $\boldsymbol{l}$ and $\boldsymbol{l}^{\prime}$ define the scattering plane (gray). The three-momentum of the virtual photon is denoted by $\boldsymbol{q}$. The angle $\phi_{S}$ is the azimuthal angle of the spin $\boldsymbol{S}$ of the fragmenting quark. Each hadron $i$ has its three-momentum $\boldsymbol{p}_{i}$, together they define the hadron plane (blue). The corresponding $\xi_{i}$ values are used for a normalization of the difference vector $\boldsymbol{R}$, i.e. $\boldsymbol{R}=\left(z_{2} \boldsymbol{p}_{1}-z_{1} \boldsymbol{p}_{2}\right) /\left(z_{1}+z_{2}\right)=\xi_{2} \boldsymbol{p}_{1}-\xi_{1} \boldsymbol{p}_{2}$. Hence $\phi_{R}$ is the azimuthal angle of $\boldsymbol{R}$ and $\boldsymbol{R}_{T}$ is its component perpendicular to $\boldsymbol{q}$.

\section{Theoretical framework and data selection}

In the SIDIS process $\mu N \rightarrow \mu^{\prime} h_{1} h_{2} X$ the incoming lepton is scattered off a transversely polarized quark inside the nucleon via the exchange of a virtual photon. The struck quark hadronizes into at least two unpolarized hadrons. For each oppositely charged hadron-pair, the quantity $\boldsymbol{R}$ is defined, i.e. their normalized relative momentum. Figure 1 shows a simplified scheme of this process. In the SIDIS cross section the angle $\phi_{R}$ between the dihadron plane and the scattering plane and the azimuthal angle of the spin of the initial quark $\phi_{S}$ appear in an azimuthal modulation as a function of $\phi_{R S}=\phi_{R}+\phi_{S}-\pi[1,2]$.

To select DIS events in general, kinematic cuts on the negative square of the four-momentum transfer $Q^{2}>1(\mathrm{GeV} / c)^{2}$, the fractional energy transfer of the muon $0.1<y<0.9$ and the hadronic invariant mass $W>5 \mathrm{GeV} / c^{2}$ were applied. The hadron-pair sample requires more selection w.r.t. the single hadron asymmetries analysis [3], of which the requirement for a vertex with at least three outgoing tracks (scattered $\mu^{+}$and two hadrons) is the most fundamental one. All possible combinations of oppositely charged hadron-pairs originating from the vertex are taken into account in the analysis. Each of these hadrons has to have a fractional energy $z>0.1$ and a Feynman $x_{F}>0.1$, to ensure that the hadrons are not produced in target fragmentation. Exclusively produced $\rho$ mesons are rejected by a cut on the missing energy $E_{\text {miss }}>3 \mathrm{GeV}$ of the hadron-pair. Finally a cut of $R_{T}>0.07 \mathrm{GeV} / c$ ensures a well defined azimuthal angle $\phi_{R}$. After all cuts the full statistics on the proton target consists of $45.5 \times 10^{6} h^{+} h^{-}$pairs, of which $28.0 \times 10^{6}$ are identified as pion-pairs. The deuteron sample consists of $4.0 \times 10^{6}$ pion-pairs out of $6.5 \times 10^{6} h^{+} h^{-}$pairs. 


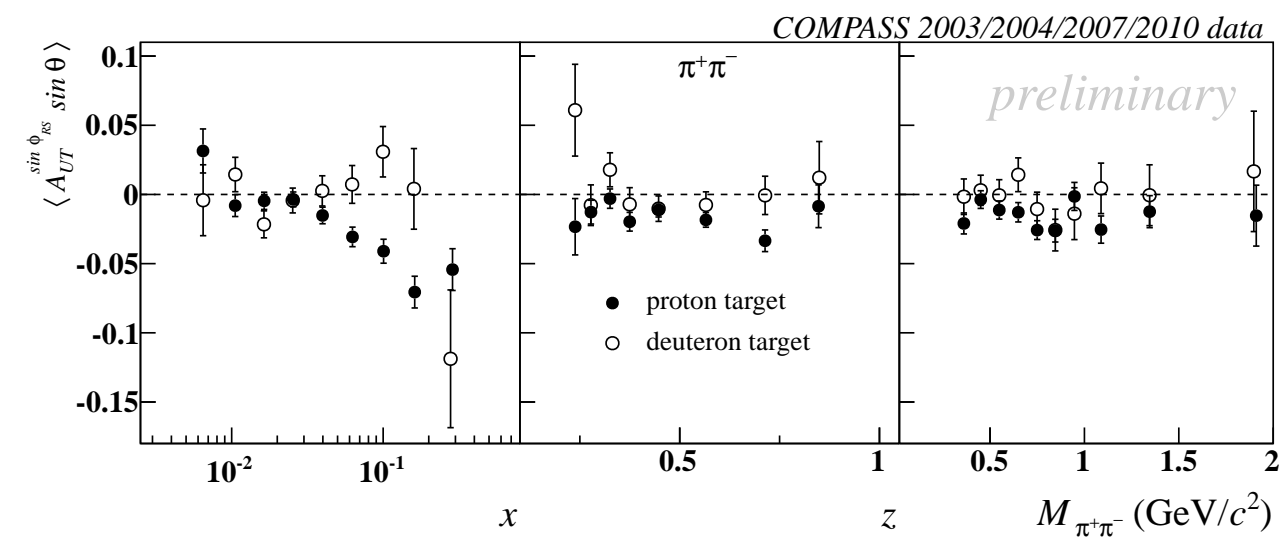

Figure 2: The identified dihadron asymmetries of $\pi^{+} \pi^{-}$from the 2003-04 deuteron data (open points) and the combined 2007/2010 proton data (dots). Only statistical uncertainties are shown.

\section{Results from the 2003-04 deuteron data}

The dihadron asymmetry of pion-pairs $\pi^{+} \pi^{-}$for the data collected in 2003-04 on the deuteron target are consistently small and compatible with zero within the uncertainties (open points in Fig. 2). Furthermore no specific trend is visible for their dependence on $x, z$ and the invariant mass of the dihadron system $M_{i n v}$. This result is in line with the COMPASS measurement of the Collins asymmetry on the deuteron [4] and the all-hadron $h^{+} h^{-}$results in [5]. This is interpreted as the consequence of a close to complete cancellation of the $u$ and $d$ quark contributions to the total Transversity distribution of the deuteron, which is also predicted by the available models $[6,7]$. The asymmetries of the other identified pair combinations $K^{+} K^{-}, \pi^{+} K^{-}$and $K^{+} \pi^{-}$are not shown here, since their are compatible with zero within their uncertainties or give only weak hints of nonzero signals.

\section{Results from the combined $2007 / 2010$ proton data}

The first measurement of the dihadron asymmetry on a proton target at COMPASS was performed using the data collected in the year 2007. The results for $h^{+} h^{-}$pairs as a function of $x, z$ and $M_{i n v}$ are presented in [5]. Recently $h^{+} h^{-}$results have been published [8], where the 2007 data has been combined with the most recent and largest data set on the proton target taken in 2010 . In both samples a large asymmetry up to $-10 \%$ in the valence $x$-region is present. As for the $z$ dependence, no specific trend is visible, while for the invariant mass a negative signal around the $\rho$ mass of $0.770 \mathrm{GeV} / c^{2}$ is observed and the asymmetry is negative over the whole mass range.

The identified pion-pair asymmetry shows a clear signal up to $-6 \%$ in $x$, the $z$ dependence is compatible with a constant and for $M_{i n v}$ a pronounced peak around the $\rho$ mass is observed. For a discussion of the other pair combinations which include kaons see Ref. [9].

The good agreement of the $\pi^{+} \pi^{-}$asymmetry amplitudes measured by COMPASS with the results obtained by the HERMES experiment [10] and also with the available model predictions [6,7] is discussed in detail in Ref. [9]. 

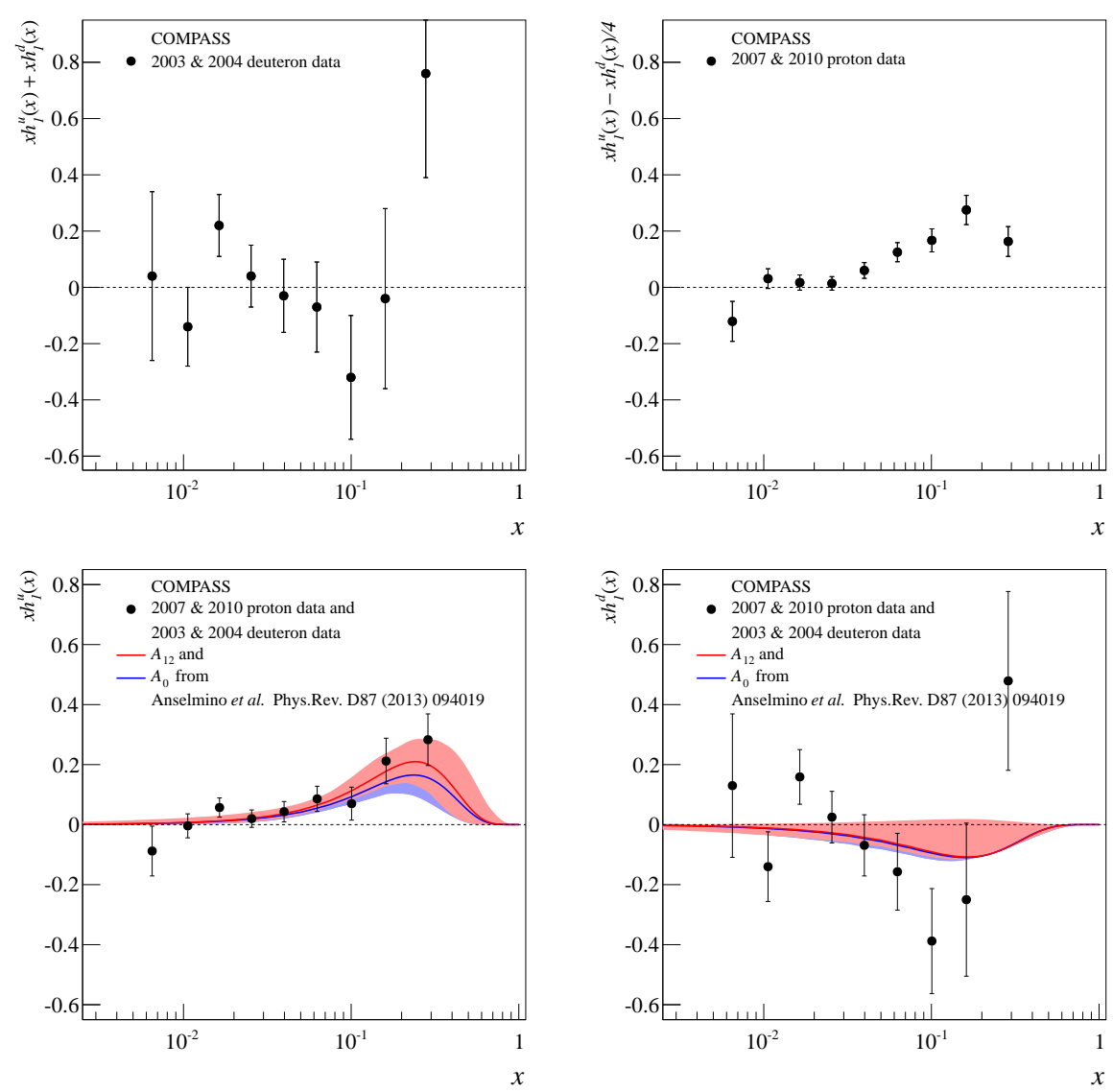

Figure 3: Results of the linear combinations $x h_{1, d}$ (upper left panel) and $x h_{1, p}$ (upper right panel) obtained from the $\pi^{+} \pi^{-}$asymmetries of the COMPASS deuteron 2003-2004 and combined proton 2007/2010 data. Point-by-point Transversity distributions for $u$ (lower left panel) and $d$ (lower right panel) valence quarks obtained from this data. They are compared to the corresponding results extracted from the single hadron Collins asymmetries by Anselmino et al. [12] using the $A_{12}$ asymmetry (red curve) and the $A_{0}$ asymmetry (blue curve) from BELLE.

\section{Extraction of the Transversity distribution and conclusions}

The complete sets of dihadron asymmetries obtained from the data taken on the transversely polarized deuteron and proton targets at the COMPASS experiment give the unique possibility to extract the Transversity distributions $x h_{1}^{q}(x)$ of $u$ and $d$ valence quarks separately, without the necessity to evolve results between different kinematic domains. The procedure executed in the following is based on the method by Bacchetta et al. [11] and their previous works, which is referenced therein.

The starting points are the linear combinations $x h_{1, p}\left(x, Q^{2}\right)=x h_{1}^{u}\left(x, Q^{2}\right)-\frac{1}{4} x h_{1}^{d}\left(x, Q^{2}\right)$ and $x h_{1, d}\left(x, Q^{2}\right)=x h_{1}^{u}\left(x, Q^{2}\right)+x h_{1}^{d}\left(x, Q^{2}\right)$, which can be extracted from the proton and the deuteron data separately. Using Table 1 of Ref. [11] the bin-by-bin ratios between $x h_{1, p / d}$ and the COMPASS dihadron asymmetries of $h^{+} h^{-}$pairs used as an input for their extraction are calculated. These ratios contain the information on the unpolarized process as well as on the polarized DiFFs $H_{1}^{\varangle q}$. They can be used as conversion factors in order to calculate the point-by-point amplitudes of the 
Transversity distribution with the new $\pi^{+} \pi^{-}$results instead the previously used $h^{+} h^{-}$results [5]. The results obtained with the new pion-pair asymmetries of the COMPASS deuteron and proton data are shown in Fig. 3 (top row). The distributions of $h_{1, p / d}$ are very similar in magnitude to the original asymmetry amplitudes, since the conversion factors are almost constant over the $x$ range. The final $u$ and $d$ valence quark Transversity distributions are obtained by solving the system of equations, namely the two linear combinations. Figure 3 (bottom row) shows the results for $x h_{1}^{u}$ and $x h_{1}^{d}$. A nonzero trend at large $x$ values is evident in both distributions, the four highest $x$ bins being at least $1 \sigma$ away from zero. From the present data, sign changes in the distribution of $x h_{1}^{d}$, for instance at very large $x$, can not be excluded due to the limited statistics. Furthermore the $d$ and $u$ quark Transversity PDFs are of opposite signs and the strength of their signals is compatible within the uncertainties. The obtained Transversity signals are also well compatible with the extraction by Anselmino et al. [12] using single hadron Collins asymmetry results and BELLE $e^{+} e^{-}$data.

\section{References}

[1] X. Artru and J. C. Collins, Measuring transverse spin correlations by 4-particle correlations in $e^{+} e^{-} \rightarrow 2$ jets, Eur. Phys. J. C 1996 (69) 277 [hep-ph/ 9504220 ].

[2] A. Bacchetta and M. Radici, Partial-wave analysis of two-hadron fragmentation functions, Phys. Rev. D 2003 (67) 094002 [hep-ph / 0212300 ].

[3] C. Adolph et al. (COMPASS Collaboration), Experimental investigation of transverse spin asymmetries in muon-p SIDIS processes: Collins asymmetries, Phys. Lett. B 2012 (717) 376 [hep-ex/1205.5121].

[4] M. Alekseev et al. (COMPASS Collaboration), Collins and Sivers asymmetries for pions and kaons in muon-deuteron DIS, Phys. Lett. B. 2009 (673) 127 [hep-ex/0802 .2160].

[5] C. Adolph et al. (COMPASS Collaboration), Transverse spin effects in hadron-pair production from semi-inclusive deep inelastic scattering, Phys. Lett. B. 2012 (713) 10 [hep-ex/1202 . 6150].

[6] A. Bacchetta and M. Radici, Modeling dihadron fragmentation functions, Phys. Rev. D 2006 (74) 114007 [hep-ph/ 0608 .037].

[7] J. She, Y. Huang, V. Barone, B.-Q. Ma, Transversity from two pion interference fragmentation, Phys. Rev. D 2008 (77) 014035 [hep-ph/ 0711 . 0817].

[8] C. Adolph et al. (COMPASS Collaboration), A high-statistics measurement of transverse spin effects in dihadron production from muon-proton semi-inclusive deep-inelastic scattering, CERN-PH-EP-2014-013 2014 [hep-ex/1401 . 7873].

[9] C. Braun (COMPASS Collaboration), COMPASS results on transverse spin dependent azimuthal asymmetries in two-hadron production in semi-inclusive deep-inelastic scattering, in proceedings of DIS2013, POS (S232) 2013.

[10] A. Airapetian et al. (HERMES Collaboration), Evidence for a Transverse Single-Spin Asymmetry in Leptoproduction of $\pi^{+} \pi^{-}$pairs, J. High Energ. Phys. 2008 (06) 066 [hep-ex/ 0803.2367 ].

[11] A. Bacchetta, A. Courtoy and M. Radici, First extraction of valence transversities in a collinear framework, JHEP 2013 (1303) 119 [hep-ph/1212 . 3568].

[12] M. Anselmino, M. Boglione, U. D’Alesio, S. Melis, F. Murgia et al., Simultaneous extraction of transversity and Collins functions from new SIDIS and $e^{+} e^{-}$data, Phys. Rev. D 2013 (87) 094019 [hep-ph/1303.3822]. 